\title{
Ureteral Access Sheath Applicatıon Without Fluoroscopy in Retrograde Intrarenal Surgery
}

\author{
Aykut Aykac ${ }^{1}$, Ozer Baran ${ }^{1}$ and Sercan Sari \\ ${ }^{1}$ Department of Urology, Faculty of Medicine, Karabuk University, Turkey \\ ${ }^{2}$ Department of Urology, Faculty of Medicine, Bozok University School of Medicine, Yozgat, Turkey
}

\begin{abstract}
Objective: To compare the results and complications of the two techniques of ureteral access sheath application, with and without using fluoroscopy.

Study Design: A comparative study.

Place and Duration of Study: Department of Urology, Karabuk University Medical Faculty Training and Research Hospital and Ankara Diskapi Yildirim Beyazid Training and Research Hospital between April 2014 and January 2018.

Methodology: Retrospective evaluation was made of patients applied with retrograde intrarenal surgery using ureteral access sheath (UAS). In Group 1, defined method were used for UAS application without using scopy. In Group 2, following semi-rigid ureterorenoscopy, localisation of guidewire was checked with fluoroscopy. UAS was advanced to ureter over guidewire under fluoroscopy imaging.

Results: Success rate of UAS placement was similar in both groups $(p=0.747)$. The time of UAS placement was 14.75 secs in Group 1 and 14.99 secs in group $2(p=0.073)$. Fluoroscopy was not used at all during UAS placement in Group 1 . In Group 2 , the mean duration of fluoroscopy use was $9.93 \pm 3.89$ secs. Total stone-free rate was $82.09 \%$ and $83.28 \%$ in Group 1 and 2 , respectively. $(p=0.653)$.

Conclusion: The method described in this paper provides protection against radiation exposure for both the patient and the operating team; and prevents potential complications by enabling clear evaluation of ureteral orifice.
\end{abstract}

Key Words: Renal stone surgery, Retrograde Intrarenal surgery, Radiation protection, Flouroscopy, Radiation free.

How to cite this article: Aykac A, Baran O, Sari S. Ureteral Access Sheath Applicatıon Without Fluoroscopy in Retrograde Intrarenal Surgery. J Coll Physicians Surg Pak 2020; 30(05):503-507. DOI: https://doi.org/10.29271/jcpsp.2020.05.503.

\section{INTRODUCTION}

Retrograde intrarenal surgery (RIRS) has become increasingly widespread in the treatment of upper urinary system stones. ${ }^{1}$ The prevalence of kidney stone disease has reached a lifetime rate of approximately $14 \% .{ }^{2}$ A systematic review from 6 countries shows that overall ureteroscopy procedure had a $251.8 \%$ increase in total number of treatments in the last decade. ${ }^{3}$ Depending on the technological development of ureteroscope and laser, RIRS is now recommended as the first-line treatment of renal stones up to $2 \mathrm{~cm}$ from EAU guidelines. ${ }^{4}$

Except ultrasonography, all the imaging methods applied in the diagnosis and follow-up of stone disease contain a certain rate of radiation. A kidney-ureter-bladder radiograph, delivers radiation exposure of 0.6-0.7 mSv, intravenous urogram delivers 3 $\mathrm{mSv}$, non-contrast abdomen computed tomography (NC- CT) exposes to14 $\mathrm{mSV} .^{5}$

Correspondence to: Aykut Aykac, Department of Urology, Faculty of Medicine, Karabuk University, Karabuk, Turkey E-mail: aykutdr@gmail.com

Received: March 19, 2020; Revised: May 15, 2020;

Accepted: May 24, 2020

DOI: https://doi.org/10.29271/jcpsp.2020.05.503
When the recurrent characteristic of stone disease is taken into consideration, the cumulative effect of radiation for patients should not be ignored. Moreover, fluoroscopy is used during placement of the ureteral access sheath in the RIRS technique or in the evaluation of stone localisation and residue within the kidney with the flexible ureteroscope, as recommended. ${ }^{4}$

The aim of this study was to evaluate the technique of RIRS without flouroscopy in terms of success rate; this will be useful for the protection of patients and the surgical team against long-term and unnecessary radiation exposure.

\section{METHODOLOGY}

A retrospective evaluation was made of patients applied with RIRS using ureteral access sheath (UAS) from two different departments - Department of Urology, Karabuk University Medical Faculty Training and Research Hospital and Ankara Diskapi Yildirim Beyazid Training and Research Hospital, Turkey between April 2014 and January 2018. Approval for the study was granted by the Local Ethics Committee of Karabuk University (Decision No: 1/26, dated: 03.01.2018) and all the procedures were applied in compliance with the principles of the Helsinki Declaration. Informed consents were obtained from all the patients.

Group 1 patients were those who applied with UAS under direct endoscopic view without the use of fluoroscopy at Karabuk University Medical Faculty Training and Research Hospital. In Group 2 
patients, UAS was applied under fluoroscopy guidance at Ankara Diskapi Yildirim Beyazid Training and Research Hospital. Patients were excluded as they were aged $<18$ years, open or laparoscopic ureteral stone surgery history, anomalous kidneys (horseshoe, pelvic, and malrotated kidneys; bifid collecting system) and history of anticoagulant agent use. Patients also were excluded from the study if a preoperative JJ stent was applied or if RIRS was performed without the use ofUAS.

Patients were evaluated in respect of demographic data, body mass index (BMI), American Society of Anaesthesiologists (ASA) score, stone localisation, and stone dimensions. Complications were evaluated using the modified Clavien classification. The duration of fluoroscopy use during UAS placement in Group 2 was measured in seconds. In both groups, the inability to place UAS was evaluated as a failure.

A detailed medical history was recorded for each patient and a systemic physical examination was made. Stone localisation and size were determined using NC-CT. Preoperative urine microscopy, urine culture, coagulation tests, and serum biochemistry evaluations were made of all the patients. Patients with bacterial production in the preoperative urine culture were treated appropriately and then underwent surgery when the urine culture was sterile. Thirty minutes preoperatively, intravenous cefazolin sodium at a dose of $2 \mathrm{~g}$ was administered as prophylaxis to all patients in both groups.

RIRS procedures were performed by 5 surgeons in Group 1 and 6 surgeons in Group 2, with minimum experience of 100 cases, for each of them. In both centres, the operations were performed under general anesthesia with the patient in the lithotomy position. After appropriate cleaning of the surgical site and draping, the ureter on the side of the stone was entered with a $6.5 \mathrm{~F}$ and $9 \mathrm{~F} \mathrm{semi--}$ rigid ureterorenoscope (Karl Storz, Germany) and a 0.035 hydrophilic guidewire were advanced to the kidney. Evaluation of ureteral pathology was made by withdrawing the semi-rigid ureterorenoscope as far as the ureteropelvic junction, and then remove leaving the guidewire in the ureter. In patients where a semi-rigid ureterorenoscope could not be applied because of the narrowness of the ureteral orifice, a JJ stent was applied and those patients were excluded from the study. In both groups, an Elite UAS (Istem Medikal, Ankara, Turkey) of internal/external diameter of 9.5/11.5 $\mathrm{F}$ and $45 \mathrm{~cm}$ length was used. The time of UAS placement measured between entrance of ureterorenoscope into the urethra to UAS placement into the ureter.

In Group 1, after placement of the hydrophilic guidewire in the ureter, the bladder was reached by entering the semi-rigid ureterorenoscope next to the guidewire. The ureterorenoscope was withdrawn from the bladderneck to be able to comfortably visualise the orifice. The UAS was advanced to the ureter over the guidewire next to the ureterorenoscope (Figure 1). The UAS entrance of the orifice were evaluated with simultaneous images on the ureterorenoscope (Figures 2 and 3). In patients where the UAS could not pass the orifice because of springing and pushing in the orifice, it could not pass into the ureter, so a JJ stent was applied and the procedure was terminated. In patients where the UAS passed the orifice comfortably, the access sheath was advanced from the orifice and monitored with images on the renoscope.

After placement of the access sheath in the ureter, the ureterorenoscope was removed from the bladder. The guidewire was removed from within the access sheath and a flexible ureterorenoscope was inserted into the UAS to place it endoscopically under the stone or UPJ. During the endoscopic view of flexible ureteroscope, the ureter lumen was dilated by irrigation and when the flexible ureteroscope was reached to the desired level, UAS was shifted through over the flexible ureteroscope and advanced in the ureterlumen.

Before starting the stone-breaking procedure, all the numbers of stones were identified in renal pelvis and calyces with flexible ureterorenoscope than checked with pre-op NC-CT. Floroscopic image was used under any suspicion of localisation of stone or residual fragments, if necessary.

In Group 2, following the semi-rigidureterorenoscopy, the localisation of the guidewire was checked with fluoroscopy. The UAS was advanced to the ureter over the guidewire under fluoroscopy imaging. When the access sheath could not be advanced or was forced, the procedure was terminated and a J stent was applied to the patient. In patients with successful placement of the access sheath in the ureter, the guidewire was removed and the procedure moved on to the flexible ureterorenoscope stage.

At the end of the procedure, the guidewire through the flexible ureteroscope is inserted into the kidney. Examination was made with flexible ureterorenoscope from the ureteropelvic junction as far as the ureteral orifice to determine the presence of ureteral damage associated with placement of the access sheath. Stonefree status was checked by Kidney-Ureter-Bladder graphy in opaque stone and USG in non-opaque stones at 4 weeks later the procedure; and if any suspicious of clinical important residual fragment checked with NC-CT. Stone-free state was defined as $<2 \mathrm{~mm}$ fragments residue.

Data obtained in the study were analysed statistically using Minitab-17 software (Minitab Inc, State College, PA, USA). Conformity of numerical data to normal distribution was evaluated with the Anderson Darling test. Descriptive data were expressed as mean \pm standard deviation (SD) values for continuous variables with normal distribution and as median (Q1-Q3) values for those with non-normal distribution Age was compared with Student's ttest. Numerical variables not showing normal distribution between the groups, such as BMI, stone size, stone density, and operation time, were compared with Mann-Whitney U-test. Relationships between categorical variables, such as gender, ASA scores, side of the stone, stone localization complication rates, postop DJ application, and stone-free rates were evaluated with Chi-square test. A value of $p<0.05$ was accepted as statistically significant.

\section{RESULTS}

The demographic characteristics and surgical results of the groups are shown in Table I.

Successful UAS placement was achieved in a total of $710(87.3 \%)$ patients, as 374 (86.9\%) patients in Group 1, and 336 (87.7\%) in Group 2. The success rate of UAS placement was similar in both groups ( $p=0.747)$. The time of UAS placement was 14.75 seconds (range, 10-20 secs) in Group 1 and 14.99 seconds (range, 10-21 secs) in Group $2(p=0.073)$. Fluoroscopy was not used at all during UAS placement in Group 1, and in Group 2, the mean duration of fluoroscopy use was $9.93 \pm 3.89$ seconds. 
Table i: Demographic features and surgical results.

\begin{tabular}{|c|c|c|c|}
\hline & Group 1 & Group 2 & p-value \\
\hline $\begin{array}{l}\text { Gender (M/F) } \\
\%(N)\end{array}$ & $\begin{array}{l}67.2(289) \\
32.8(141) \\
\end{array}$ & $\begin{array}{l}71(272) \\
29(111)\end{array}$ & $0.241 *$ \\
\hline Age (years) mean $\pm S D$ & $46.6 \pm 13.11$ & $45.4 \pm 13.01$ & $0.155^{\phi}$ \\
\hline BMI median (min-max) & $25(18-45)$ & $26(18-44)$ & $0.705 \dagger$ \\
\hline $\begin{array}{l}\text { ASA N } \\
1 \\
2 \\
3\end{array}$ & $\begin{array}{c}51 \\
287 \\
92 \\
\end{array}$ & $\begin{array}{c}50 \\
248 \\
85\end{array}$ & $0.877^{*}$ \\
\hline $\begin{array}{l}\text { Stone side (right/left) } \\
\%(N)\end{array}$ & $\begin{array}{l}46.5(200) \\
53.5(230)\end{array}$ & $\begin{array}{l}52.7(202) \\
47.3(181)\end{array}$ & $0.076 *$ \\
\hline $\begin{array}{l}\text { Stone location \% (N) } \\
\text { Upper ureter } \\
\text { Renal }\end{array}$ & $\begin{array}{l}30.2(130) \\
69.8(300)\end{array}$ & $\begin{array}{l}33.4(127) \\
66.6(253)\end{array}$ & $0.370 *$ \\
\hline $\begin{array}{l}\text { Stone size median } \\
\text { (min-max) }\end{array}$ & $\begin{array}{c}15.5(5-64) \\
\mathrm{mm}\end{array}$ & $\begin{array}{c}15.7(5-62) \\
\mathrm{mm}\end{array}$ & $0.926 \dagger$ \\
\hline $\begin{array}{l}\text { Dansity (Hounsfield unite) } \\
\text { median (min-max) }\end{array}$ & $\begin{array}{l}1084 \pm 399 \\
(387-1867)\end{array}$ & $\begin{array}{l}1073 \pm 340 \\
(393-1789)\end{array}$ & $0.873 \dagger$ \\
\hline Postop J stent & $72.79 \%$ & $75.97 \%$ & $0.299 *$ \\
\hline $\begin{array}{l}\text { Flouroscopy time (min) } \\
\text { Mean } \pm \text { SD }\end{array}$ & 0 & $9.93 \pm 3.89$ & \\
\hline $\begin{array}{l}\text { Operation time }(\mathrm{min}) \\
\text { Mean } \pm \mathrm{SD}\end{array}$ & $44.68 \pm 14.03$ & $45.92 \pm 14.28$ & $0.116 \dagger$ \\
\hline Stone free & $82.09 \%$ & $83.28 \%$ & $0.653^{*}$ \\
\hline
\end{tabular}

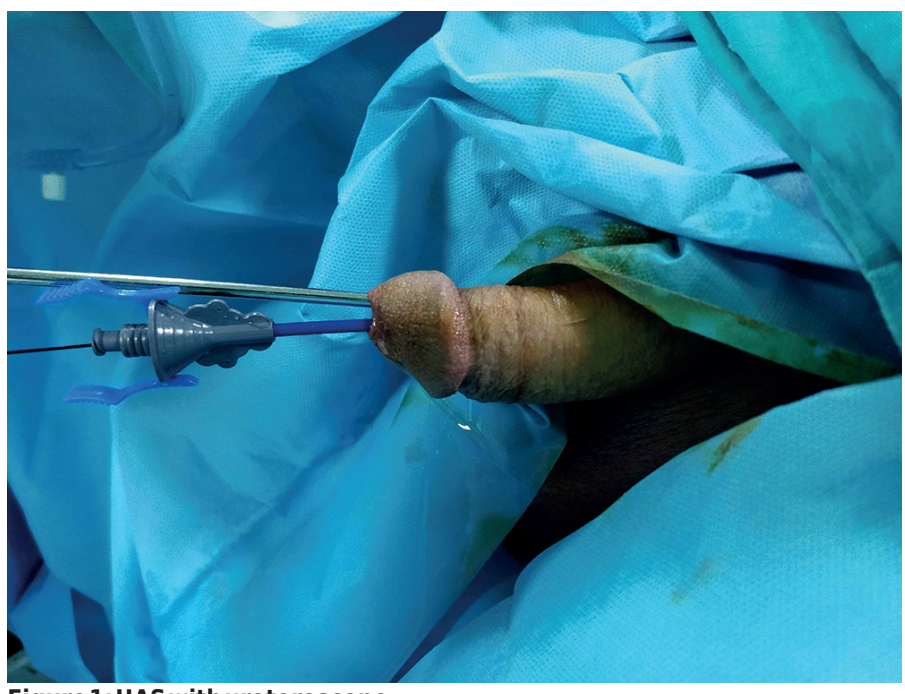

Figure 1: UAS with ureteroscope.

Clavien grade 1-2 complications associated with UAS placement were observed in 17 (3.9\%) patients (9 hematuria, 5 fever, 3 postobstructive diureses) in Group 1 and in 13 (3.3\%) patients (10 hematuria, 3 fever) in Group 2. There was no blood transfusion required for hematuria patients, all of them resolved with iv hydration. No grade 3-5 complications were observed in either group.

\section{DISCUSSION}

Radiation has deterministic and stochastic effects. The deterministic effect is occurred when exceeding the threshold radiation level. Stochastic effect is radiation dose-related rather than the severity of the damage incurred and do not have any threshold level. Stochastic effect is responsible for cancer. ${ }^{6}$ Radiation induces carcinogenetic mutations. The risk of mutations increases with the dose of radiation. The effects of radiation in the early period of exposure may not be immediately visible. Certain types of cancer may occur in the late period after radiation exposure. This effect can develop for leukemia in five years, while solid organ tumors may remain latent for 20 years. Cancer-induced damage may develop in one of 1000 patients. The use of fluoroscopy can increase the total yearly effective radiation dose and pose potentiallong-term risks. ${ }^{7}$

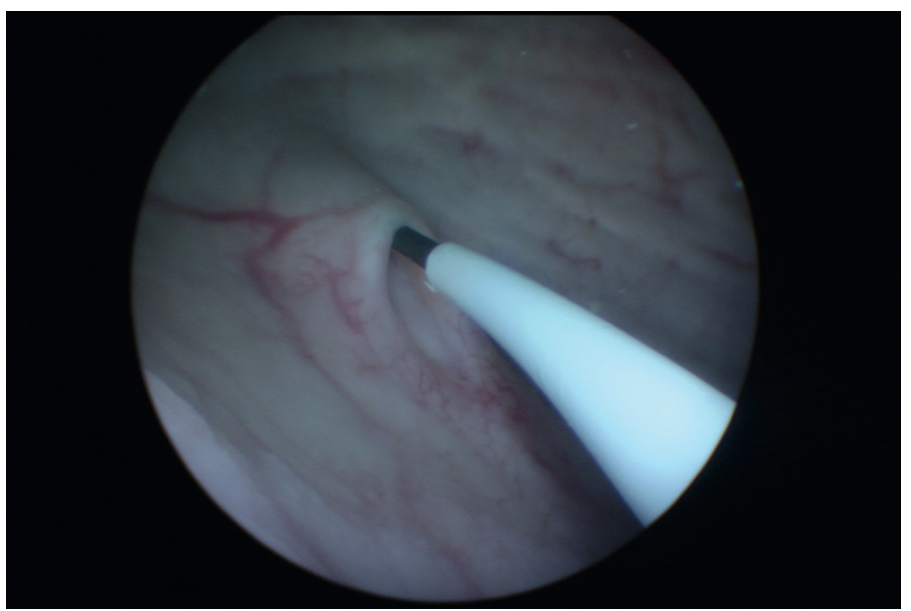

Figure 2: Observation of UAS entry into orifice.

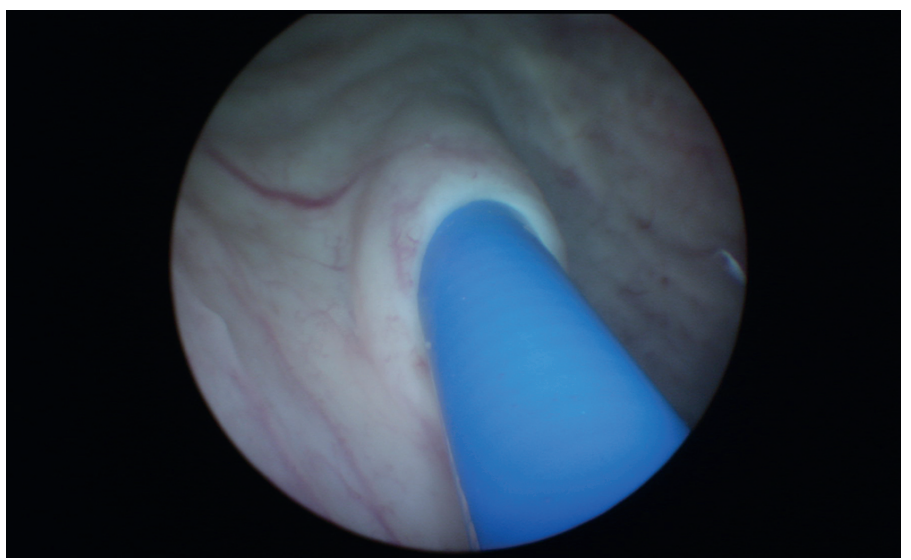

Figure 3: Observation of UAS entry into orifice.

Imaging methods are applied in the diagnosis and follow-up of urinary system stone disease. Due to the recurrent nature of stone disease, patients are radiographically evaluated at regular intervals. Ferrandino et al. ${ }^{8}$ reported that after an acute stone event, patients are examined radiographically on average 4 times within a year and approximately $20 \%$ of patients receive more than 50 $\mathrm{mSv}$ radiation. The International Commission on Radiological Protection (ICRP) has reported that exposure of $50 \mathrm{mSv}$ per year or 20 mSV per year in a 5-year period should be avoided. ${ }^{9}$ Nevertheless, in the first year offollow-up, 17-20\% of stone disease patients are exposed to $>50 \mathrm{mSv}$ radiation. ${ }^{10}$ In the USA, it has been estimated that there is a cumulative cancer risk of $0.4 \%-0.9 \%$ from radiation during diagnostic methods. ${ }^{7,11}$

Patients are exposed to radiation not only during diagnosis of stone disease but also during treatment. Depending on the treatment method applied, there is the question of radiation exposure at varying rates. During flexible ureteroscopy, the use of fluoroscopy can be required at certain stages of the operation. Fluoros- 
copy is routinely used in the placement of UAS and to be able to localise the stone within the kidney. During ureteroscopy, patients may be exposed to $2-100 \mathrm{mSv}$ radiation. ${ }^{12}$ In a cadaver organ-specific radiation model mimicking the use of fluoroscopy during ureteroscopy, Krupp et al. ${ }^{7}$ determined the risk of cancer development in 1/1000 patients associated with cellular damage triggered by radiation.

During the treatment of stone disease, it is not only the patient who is exposed to radiation but also the operating team. Hellawell et al. determined that urologists receive mean $11.6 \mu \mathrm{Gy}$ radiation per case. Although this is an extremely low dose, a surgeon performing 500 cases per year is exposed to approximately 5.8 $m G y$ radiation. This dose is almost half as much again as the dose received in $\mathrm{NC}-\mathrm{CT}{ }^{13}$ Furthermore, this dose is only that which is received during flexible ureteroscopy and there are publications that have reported much higher doses of exposure during percutaneous nephrolithotomy (PNL). ${ }^{14}$ Soylemez et al. ${ }^{15}$ reported that $96 \%$ of urologists prefer fluoroscopy-assisted PNL and only $46 \%$ always used a thyroid protector. When these conditions are taken into consideration, the application of a procedure without fluoroscopy constitutes a great advantage.

Radiation should be avoided by both the patient and the operating team. There are reports in literature that the surgical procedure can be applied without the use of fluoroscopy during flexible ureteroscopy. ${ }^{16-18}$ The use of fluoroscopy during flexible ureteroscopy is a particular point in the placement of the UAS. Decreasing the intrarenal pressure, enabling multiple access to pelvicalyceal system, improving visibility and protecting the flexible ureteroscopy, are some advantages of using UAS during RIRS. ${ }^{19}$ In the surveys conducted among the members of the Endourology Association, the routine UAS usage rate was $58 \%$ in 2015 , and increase to $75 \%$ in $2019 .{ }^{20,21}$

Various approaches have been defined for the placement of UAS without the use of fluoroscopy. In a series of 76 cases using tactile senses during UAS placement and single-shot fluoroscopy to check the placement, it was reported that 5.27 secs fluoroscopy use was required in only 4 patients. ${ }^{22}$ In the current study, the duration of fluoroscopy was measured as 6.6 secs in group 2 where it was used; and this was found to be similar to findings in literature. Also, successful UAS placement with tactile senses has been reported in pediatric patients. ${ }^{23}$ Another method that has been described in English literature is the procedure in which a 10F UAS is placed over a $7.5 \mathrm{~F}$ semi-rigid ureteroscope for placement at the ureteropelvic level during ureteroscopy without the use offluoroscopy. In a study which compared this procedure with a group where fluoroscopy was used, no statistical difference was determined in the success rates at 1 month. ${ }^{16}$ In a study of 140 selected patients by Peng et al. ${ }^{24}$, the UAS was placed over the guidewire by measuring the distance between the ureteropelvic junction and the urethral mea during diagnostic ureteroscopy, and with the exception of one patient with a double collecting system, flexible ureteroscopy was applied without fluoroscopy.

Unlike previous reports in literature, the method described in this paper provides clear visualisation of the passage of the UAS from the ureteral orifice and in cases with a narrow ureteral orifice, the movement of the orifice cranially when forcing the UAS can be clearly observed. Observation of the ureter orifice provides addi- tional contribution to the control of the force you apply in the UAS application. Provides the possibility of limiting the power application that you cannot see in fluoroscopy, if spring or thrust is occurred in the ureter orifice.

The ureter dilatation was made by semi-rigid ureteroscope at first; and by the irrigation during the flexible ureteroscope, secondly. We use the smallest size of the UAS at the market which is 9,5-11,5 Fr. UAS was shifted through over the flexible ureteroscope and advance the ureter lumen where we desired. Although theoretically the possibility of intussusception in the ureter is considered due to the difference in diameter between the access sheath and the flexible ureteroscope, this probability decreases considerably as a result of ureter dilatation. We would like to state that we have not encountered such a complication in our own series. Similar application was performed by Ekici and colleague ${ }^{25}$, and they reported only minor complications as hematuria and fever.

In addition, as in UAS placement over the ureteroscope as described in literature, our technique can be made without the need for a 7.5Fr ureteroscope. All sizes of UAS can be applied in this way without the need for an additional instrument. Ureteroscopy applied without fluoroscopy also avoids the restricted movement of the surgeon associated with the lead apron and the fatigue created over time by the weight of the lead apron. As there is no radiation exposure, this can even be safely applied to pregnant patients. Pediatric patients also avoid radiation. ${ }^{23}$

To the best of authors' knowledge, the method applied in this study is herein described for the first time in English literature. The retrospective design of the study could also be considered a limitation.

\section{CONCLUSION}

The method described in this paper can be considered of importance in respect of providing protection for the patient and the operating team against fluoroscopy exposure and in preventing potential complications by clearly evaluating the ureteral orifice.

\section{ETHICAL APPROVAL:}

Approval for the study was granted by the Local Ethics Committee of Karabuk University (Decision No: 1/26, dated: 03.01.2018) prior to initiation of the research work.

\section{PATIENTS' CONSENT:}

Informed consents were obtained from all patients to publish the data concerning this case.

\section{CONFLICT OF INTEREST:}

Authors declared no conflict of interest.

\section{AUTHORS' CONTRIBUTION:}

AA: Manuscript writing, acquisition, conception of idea and final approval.

OB: Interpretation of data, data collection and analysis, critical review.

SS: Design of the work, intellectual content, supervision.

\section{REFERENCES}

1. Oberlin DT, Flum AS, Bachrach L, Matulewicz R, Flury SC. Contemporary surgical trends in the management of upper tract calculi. J Urol 2015; 93(3):880-4. 
2. Rodriguez-Monsalve Herrero M, Doizi S, Keller EX, Coninc VD, Traxer O. Retrograde intrarenal surgery: An expanding role in treatment of urolithiasis. Asian J Urol 2018; 5(4):264-73.

3. Geraghty RM, Jones P, Somani BK. Worldwide trends of urinary stone disease treatment over the last two decades: A systematic review. J Endourol 2017; 31(6):547-56.

4. Turk C, Petrik A, Sarica K, Christian Seitz, Andreas Skolarikos, Michael Straub, et al. EAU guidelines on interventional treatment for urolithiasis. Eur Urol 2016; 69(3):475-82.

5. Mettler FA Jr, Huda W, Yoshizumi TT, Mahesh M. Effective doses in radiology and diagnostic nuclear medicine: $A$ catalog. Radiology 2008; 248(1):254-63.

6. Chen $T T$, Wang $C$, Ferrandino MN, Scales CD, Yoshizumi $T T$, Preminger GM, et al. Radiation exposure during the evaluation and management of nephrolithiasis. J Urol 2015; 194(4):878-85.

7. Krupp N, Bowman R, Tenggardjaja C, Jellison F, Hill $B$, Ebrahimi $\mathrm{K}$, et al. Fluoroscopic organ and tissue-specific radiation exposure by sex and body mass index during ureteroscopy. J Endourol 2010; 24(7):1067-72.

8. Ferrandino MN, Bagrodia A, Pierre SA, Charles D Scales Jr, Rampersaud $\mathrm{E}$, Margaret $\mathrm{S}$, et al. Radiation exposure in the acute and short-term management of urolithiasis at 2 academic centers. J Urol 2009; 181(2):668-72 discussion 673.

9. 1990 Recommendations of the international commission on radiological protection. Ann ICRP 1991; 21(1-3):1-201.

10. Elkoushy MA, Andonian S. Lifetime radiation exposure in patients with recurrent nephrolithiasis. Curr Urol Rep 2017; 18(11):85

11. Perisinakis $K$, Damilakis J, Anezinis $P$, Tzagaraki $L$, Varveris $H$, Cranidis $A$, et al. Assessment of patient effective radiation dose and associated radiogenic risk from extracorporeal shock-wave lithotripsy. Health Phys 2002; 83(6):847-53.

12. Bagley DH, Cubler-Goodman A. Radiation exposure during ureteroscopy. J Urol 1990; 144(6):1356-8.

13. Hellawell GO, Mutch SJ, Thevendran G, Wells E, Morga RJ. Radiation exposure and the urologist: What are the risks? J Urol 2005; 174(3):948-52; discussion 952.

14. Demirci A, Raif Karabacak O, Yalcinkaya F, Yiğitbaşı O, Aktaş C. Radiation exposure of patient and surgeon in minimally invasive kidney stone surgery. Prog Urol 2016; 26(6):353-9.

15. Soylemez H, Altunoluk B, Bozkurt Y, Sancaktutar AA, Penbegül N, Atar M. Radiation exposure-do urologists take it seriously in Turkey? J Urol 2012; 187(4):1301-5.

16. Karabulut I, Keskin E, Bedir F, Ziypak T, Doluoglu OG, Resorlu B, et al. Rigid ureteroscope aided insertion of ureteral access sheath in retrograde intrarenal surgery. Urology 2016; 91:222-5.

17. Cimen HI, Halis F, Saglam HS. Flouroscopy-free technique is safe and feasible in retrograde intrarenal surgery for renal stones. Turk J Urol 2017; 43(3):309-12.

18. Hsi RS, Harper JD. Fluoroless ureteroscopy: Zero-dose fluoroscopy during ureteroscopic treatment of urinary-tract calculi. J Endourol 2013; 27(4):432-7.

19. Kaplan AG, Lipkin ME, Scales CD Jr, Preminger GM. Use of ureteral access sheaths in ureteroscopy. Nat Rev Urol 2016; 13(3):135-40.

20. Dauw CA, Simeon L, Alruwaily AF, Sanguedolce F, Hollingsworth JM, Roberts WW, et al. Contemporary practice patterns of flexible ureteroscopy for treating renal stones: Results of a worldwide survey. J Endourol 2015; 29(11):1221-30.

21. Zilberman DE, Lazarovich A, Winkler H, Kleinmann N. Practice patterns of ureteral access sheath during ureteroscopy for nephrolithiasis: A survey among endourologists worldwide. BMC Urol 2019; 19(1):58.

22. Kirac M, Tepeler A, Guneri C, Kalkan S, KArdas S, Abdullah A, et al. Reduced radiation fluoroscopy protocol during retrograde intrarenal surgery for the treatment of kidney stones. Urol J 2014; 11(3):1589-94.

23. Kirac M, Ergin G, Kibar $Y$, Köprü B, Biri H. The efficacy of ureteroscopy without fluoroscopy for ureteral and renal stones in pediatric patients. J Endourol 2018; 32(2):100-5.

24. Peng $Y, X u B$, Zhang $W$, Li L, Liu M, Gao X, et al. Retrograde intrarenal surgery for the treatment of renal stones: Is fluoroscopy-free technique achievable? Urolithiasis 2015; 43(3): 265-70.

25. Ekici M, Ozgur BC, Senturk AB, Ekici A A, Aydin C, Baykam $M M$. All seeing-access sheath: A novel fluoroscopy-free placement technique in retrograde intrarenal surgery. J Coll Physicians Surg Pak 2019; 29(3):263-7. 